\title{
Математическое моделирование трехстороннего рынка: медиа, производство и потребители
}

\section{Сергей Вартанов}

Московский государственный университет имени М.В. Ломоносова, Москва, Россия

\author{
Информация о статье \\ Поступила в редакичию: \\ 10.04.2020 \\ Принята \\ к опубликованию: \\ 04.06.2020
}

УДК 51-77

JEL C51, C52

\begin{abstract}
Ключевые слова:
модели двустороннего рынка, экономика рекламы, экономика медиаиндустрии, несовершенная конкуренция, медиарекламный рынок, математическое моделирование
\end{abstract}

\begin{abstract}
Keywords:
two-sided market model, advertising economy, media industry economy, imperfect competition, mediaadvertising market, mathematical modelling
\end{abstract}

\begin{abstract}
Аннотация
В работе формулируется общая методология построения моделей трехстороннего рынка, возникающего при неразделимом влиянии друг на друга медиа, производства и потребления, а также рассматриваются два примера таких моделей: модель рынка двусторонней монополии, на котором имеется один потребитель, одна фирмапроизводитель товара и одна медиафирма, и модель рынка горизонтально дифференцированного товара и контента. Для этих примеров исследуются свойства рыночных равновесий и их качественных особенностей. Кроме того, формулируются основные направления дальнейшего развития моделей трехсторонних рынков как отдельного раздела математической экономики.
\end{abstract} Mathematical Modeling of the Three-Sided Market: Media,
Production and Consumers

Sergey Vartanov

\begin{abstract}
The article considers the issue of mathematical modelling of the media intersectoral influence on the other branches of economy. There are some ways of this influence, one of the most prominent among them is the advertising. There are plenty of works relating to the influence of advertising on the stated economic subjects and on the market balance in general. Although there are a lot of studies on these issues, the unified theory based on the synthesis of advertising and production models, as well as consumer choice models, does not yet exist. This leads to the second objective of the this paper: to analyze the white spots in the modern theories and to determine the most promising directions for the economic theory development taking due account of advertising and its place in the structure of the economy, as well as to raise the question of constructing a new class of economic models considering the media economic segment as an integral part of each market. Some examples of these threesided market models are considered in this paper.
\end{abstract}




\section{Введение}

Анализ потребностей современной аудитории медиа показывает, что современный человек использует медиа для удовлетворения тех же потребностей, что и экономические блага [1]. Потребление экономических благ и контента, предлагаемого средствами массовой коммуникации и медиакомпаниями, происходит не только одновременно, но и с одними целями, связанными с удовлетворением базовых человеческих потребностей [2]. С точки зрения экономики это означает, что и товары, и контент медиа являются равноправными благами, совместный выбор которых представляет собой единую задачу потребителя в современной цифровой экономике. Разумеется, в наибольшей степени это характерно для молодежи, однако и среди представителей иных поколений это становится справедливым (пусть и в меньшей степени).

Воздействие медиа на структуру и исход взаимодействия участников любого рынка значительно и многопланово, его эффекты проявляются на нескольких уровнях. Безусловно, стремительная трансформации медиа под влиянием цифровизации изменила многие принципы функционирования медиа не только как индустрии, но и как части глобального информационного контекста. Несмотря на эту трансформацию, ключевым механизмом влияния медиа на производство и потребление экономических благ является реклама. Будучи особым типом медиаконтента, она представляет собой часть информационного наполнения медиа, потребление которого происходит параллельно с потреблением «обычного» контента. Хотя для потребителя сравнимой полезности она не приносит, под влиянием рекламы изменяется выбор потребителем экономических благ. Это делает рекламу, размещаемую медиакомпаниями в контенте связанных с ними средств массовой коммуникации, эффективным способом манипулирования спросом, широко используемым фирмами-производителями товаров и экономических благ. Таким образом, медиафирмы, потребители-аудитория и производство экономических благ оказываются неразрывно связаны, образуя своего рода треугольник. Он и представляет собой трехсторонний рынок, в котором все стороны рынка оказывают друг на друга существенное влияние, экономическим эффектами которого невозможно пренебречь.

\section{Теоретико-экономические рамки исследования взаимовлияния медиа, производства и потребления \\ В экономической теории можно обнаружить два основных подхода} (идеологических лагеря), используемых для анализа взаимного влияния медиа и производства экономических благ. Первый связан с всесторонним анализом влияния рекламы как одного из компонентов рыночной стратегии производителей на структуру рынка их продукции. В моделях, описываемых в работах этой группы, основными субъектами рынка являются потребители и производители - как и в классических моделях рыночного равновесия, а роль медиа сведена до чисто инструментальной. Вторая группа концентрируется на моделировании непосредственно рынка медиа, в частности, конкуренции между медиафирмами за аудиторию и рекламодателей, которые, в свою очередь, представляются в виде двух групп потребителей услуг, предлагаемых медиа. 
Существующая теоретическая литература, посвященная исследованию и моделированию рынков с возможностью рекламного воздействия может быть условно разделена на три основных направления. Эти направления связаны с базовыми вопросами экономической теории рекламы, посвященными ее влиянию на субъекты рынка, свойства рыночного равновесия и структуру рынка в целом. Первое направление основано на вопросах, касающихся оценки влияния рекламы на поведение потребителей: как меняется способ принятия экономических решений и к каким изменениям в спросе он приводит. Вопросы, анализируемые в работах второго направления, связаны с поведением рекламодателей - фирм и посредников: что можно считать «рекламной стратегией фирмы», как эти стратегии позволяют фирме «управлять» спросом и какие из них можно считать оптимальными в зависимости от особенностей товара, возможностей фирмы и т.п. Третья группа вопросов связана с влиянием рекламы как экономического института на рыночное равновесие: выигрывает ли общество от появления в руках фирм рекламных инструментов, какие участники рынка выигрывают, а какие проигрывают из-за этого.

Описанные вопросы хорошо изучены в литературе. Для любого типа рекламного воздействия известно, как определить совокупный спрос как функцию параметров этого воздействия [3, 4]. На основании этой функции фирмы находят оптимальный вид стратегии продвижения своего товара (например, пульсирующий либо поддерживающий $[5,6])$. От устанавливающегося в этом случае рыночного равновесия могут выиграть различные игроки. Например, на монопольном рынке от появления рекламы выигрывает только производитель, а вот общественное благосостояние снизится [3]. В то же время, на олигопольных рынках реклама может оказывать позитивное влияние и на излишек потребителя, и на прибыль производителей (например, создавая барьер для входа новых фирм на рынок) [7, 8].

Математические модели рыночного равновесия достаточно хорошо изучены, достаточно вспомнить классические концепции равновесия Вальраса для совершенно конкурентной экономики, равновесия Курно и Бертрана для олигополии и т.д. Все они основаны на том, что на рынке существуют два типа агентов - потребители и производители. Первые выбирают оптимальный набор товаров, вторые - оптимальные планы производства, максимизирующие прибыль. Однако эти модели построены на базовом предположении о рациональности субъектов. Рациональность означает, что предпочтения потребителей непротиворечивы и не зависят от контекста принятия решения, однако данное предположение выполняется далеко не всегда. Манипуляция контекстом принятия решений возможна: рекламное убеждение, искусственное и естественное ограничение информации, специальное конструирование множества выбора и так далее. При принятии потребителем решений особую важность имеет информация, определяющая контекст этого принятия. Например, информативная реклама не влияет на ценовую эластичность совокупного спроса, чего нельзя сказать об убеждающей: чем интенсивнее продвигается какой-либо товар, тем менее эластичным по цене становится спрос на него.

Для основных типов рекламного воздействия на потребителя можно построить соответствующую функцию совокупного спроса, отражающую качественные особенности влияния такого воздействия на спрос. Совокуп- 
ный спрос потребителей зависит от интенсивности рекламного воздействия, определяемого в значительной мере затратами фирмы на рекламу своего товара, а также структурой их распределения по различным типам рекламы и медиаканалам. Когда фирма понимает, как ее рекламные затраты сказываются на совокупном спросе, она получает возможность планировать одновременно и ценовую, и рекламно-информационную стратегию для максимизации совокупной прибыли. Однако в реальности рекламировать товары могут не только их производители, но и последующие единицы в аффилированных с ними производственных цепочках [9]. Появление между производителями и потребителями посредников, управляющих рекламным воздействием первых на спрос последних, приводят к возникновению производственных цепочек особого рода - маркетинговых каналов.

Традиционно рассматриваемые в теории организации рынков маркетинговые каналы включают в себя производителей и продавцов-ритейлеров [10]. В то же время на исход рыночного взаимодействия влияют и другие субъекты, связанные только с каналами доставки информации. Например, фирмы, владеющие средствами массовой коммуникации, в контенте которых размещается реклама. Телеканалы, интернет-порталы и радиостанции являются участниками рынка, которые фактически «продают» свою аудиторию производителям. При моделировании их поведения в существующей литературе применяются модели двусторонних рынков [11]. Такое название закрепилось за классом моделей рынков особого типа, на которых продавец взаимодействует с покупателями, разделенными на две группы (например, аудитория и рекламодатели). Он реализует каждой группе покупателей разные товары, удовлетворяющие принципиально различные потребности, однако спрос одной группы существенно зависит от спроса второй группы, и наоборот. Роль производителя на данном рынке - быть платформой, соединяющей две группы потребителей.

Медиарынки, очевидно, можно отнести к рынкам подобного типа: платформой на них выступает медиа, которое объединяет две группы пользователей: аудиторию средств массовой коммуникации, покупающую контент у фирм-владельцев медиаканалов (медиафирм), и рекламодателей, покупающих у них же рекламные контакты с аудиторией [12]. Под контентом при этом понимается информационное наполнение данного медиа. Например, для телеканала контентом являются транслируемые на нем программы и кинофильмы, для печатных СМИ - статьи, для интернет-сайтов - публикуемые там материалы любого рода и т.д. Очевидно, что спрос потребителей двух типов на услуги медиа существенно взаимозависим. Спрос на размещение рекламы в медиа тем выше, чем большую аудиторию сможет привлечь к себе медиа; с другой стороны - зрители и слушатели медианосителя при изменении объема рекламы в СМИ могут изменять объем потребления этого носителя [13]. В подобной постановке экономическое воздействие рекламы описывается как возникающие в модели двустороннего рынка сетевые эффекты [14].

Таким образом, средства массовой коммуникации являются полноправными участниками любого рынка, допускающего рекламное воздействие производителей на спрос. Эти участники (медиафирмы) производят и прода- 
ют товар двух типов: аудиторию производителям, а контент - аудитории. Это порождает задачу соединения моделей рынков классических товаров с рекламой и моделей медиарынков, где реклама - не метод воздействия на спрос потребителей, а полноценный товар. Медиафирмы в двусторонних моделях обладают стабильной структурой аудитории, их стратегией является выбор цен на рекламу, в случае двойного финансирования - еще и меню предлагаемого потребителям контента (то есть его типов и их цен для потребителя). Прибыль медиафирм складывается из выплат от рекламодателей контрактов и, если медиафирма производит и продает контент, то дополнительно и от его потребителей. Выплаты от рекламодателей определяются долей потребителей, которых удастся охватить показами рекламы их товара. Воздействие рекламы предполагается строго информирующим, потребитель, столкнувшийся с рекламой, лишь узнает о существовании товара, но не меняет своих предпочтений. Потребитель выбирает медиаканалы, с которыми хотел бы контактировать, а его выигрыш определяется как разность полезности от использования медиасервисов (контента, коммуникативных возможностей социальных сетей) и негативного воздействия, связанного с раздражением потребителя от избытка рекламы. Важно отметить, что реклама фирм «внедряется» в контент, производимый медиафирмами: телепрограммы «перемежаются» рекламными блоками, на газетных полосах между статьями вставляются рекламные объявления, на интернет-сайтах рекламные баннеры могут находиться посреди текста, как и контекстная реклама. Потребитель выбирает те медиаканалы, с которыми хотел бы контактировать, максимизируя полезность в виде разности двух показателей - собственно полезности от потребления контента медиа [15] или коммуникативных возможностей социальных сетей [16] и негативного воздействия, связанного с раздражением потребителя от избытка рекламы (advertising nuisance [17]).

Рекламодатели в двусторонних моделях рынка представляют собой особый тип потребителей, отличных от аудитории. Задача рекламодателя заключается в определении оптимального набора медиаканалов и объемов этой рекламы. Критерием оптимальности является полезность, определяемая как разность вида $\psi(\vec{y})=A(\vec{y})-G(\vec{y})$, где $\vec{y}-$ вектор, составленный из объемов рекламы в каждом из существующих медиа, $A(\cdot)$ - влияние такого рекламного воздействия на ожидаемую прибыль рекламодателя, а $G(\cdot)$ - затраты на размещение рекламы. Основным вопросом, на который отвечают двусторонние модели медиарынков, является вопрос ценообразования на размещение рекламы в медиа и на контент. Ответ на него, естественно, существенно зависит от структуры медиарынка. Например, если существует всего одна медиафирма, то она склонна занижать цены на контент (по сравнению с немедийным монополистом, работающим на одностороннем рынке), так как это стимулирует спрос на контент и позволяет повысить цены на размещение рекламы. В то же время, для контента справедлива на первый взгляд контринтуитивная особенность: появление на медиарынке второй фирмы может не снизить, а повысить цену на контент. Это может произойти тогда, когда конкуренция на рынке контента невысока: за счет повышения цены на него медиафирмы компенсируют потери от обострения конкуренции за рекламодателей. 
Примером такого рынка является рынок с двумя телеканалами - новостным и кинематографическим, взаимозаменяемость двух типов контента (новостей и фильмов) на котором довольно низка. Итоговое соотношение монопольной и конкурентной цен на контент зависит от соотношения между двумя разнонаправленными эффектами: «традиционного» снижения цены за счет усиления конкуренции и «повышающего» эффекта, возникающего из-за того, что меньшей цене на контент соответствует больший спрос на него и, соответственно, меньшее значение предельной рекламной выручки за один контакт. В то же время монопольная цена за размещение рекламы соответствует ожидаемым предположениям и оказывается выше олигопольной, так как на стороне рекламодателей «повышающего» эффекта не возникает [15].

\section{Трехсторонние модели: концептуализация и математическая постановка}

Двусторонние модели хорошо описывают медиарынок, учитывая многие его особенности. Тем не менее, ряд существенных моментов они упускают. Например, многим рекламодателям важна не реклама сама по себе и не количество контактов, а те убеждающие и информативные эффекты, которые они окажут на спрос. Потребители, в свою очередь, выбирают не только контент, но и товары для потребления. Эти товары производятся рекламодателями, которые для медиафирм - лишь «другие» потребители. Подобные недостатки связаны с тем, что каждый из существующих подходов не учитывает полноценно какую-либо из сторон рыночного взаимодействия в условиях информационной экономики:

- модели рекламного равновесия не учитывают субъектность медиа;

- двусторонние модели не учитывают производственный характер рекламодателей.

В отличие от двусторонней модели медиарынка, в трехсторонней модели одни и те же потребители выступают одновременно в двух «ролях»- потребителей товара и потребителей медиаконтента. При этом потребление медиаконтента (как и в двусторонних моделях) приносит им полезность, как и потребление «обычных» благ (например, просмотр кино по телевизору или чтение статьи в журнале [1]). Особым типом контента является и сама реклама: она представляет собой часть информационного наполнения медиа. Однако полезности от потребления, сравнимой с «полноценным» медиапотреблением, она не приносит, лишь изменяет выбор потребителем экономических благ (информируя об их свойствах, изменяя полезность от их потребления). В то же время потребление рекламы происходит параллельно с потреблением «обычного» контента. Например, тратя два часа на просмотр фильма по телевизору, примерно пятнадцать минут телезритель смотрит рекламные паузы, из двухсот страниц модного журнала примерно половина приходится на рекламу, полностью исключить контакт с которой читателю невозможно. «Внедренные» подобным образом в медиаконтент рекламные сообщения (в том числе и спонсорский контент [18]) дают производителям товаров возможность производить рекламное убеждение, изменяющее полезность от потребления немедийных благ. Фирмы-владельцы СМИ представляют собой 
третий тип участников рынка, отличающийся как от производителей, так и потребителей - они создают контент, продавая его потребителям, и доводят до них рекламу производителей, «продавая» им свою аудиторию. При этом доходы этих фирм включают доходы как от продажи рекламы [19], так и от реализации контента $[15,16]$.

Приведем формулировку трехсторонней модели для весьма общего случая: все фирмы на рынке (и производители, и медиа) являются олигополистами типа Курно на своих рынках - то есть «рыночная» часть их стратегий связана с выбором объемов производства. Естественно, рынки могут быть устроены и иначе, примеры таких моделей рассмотрены в настоящей работе ниже. Например, медиарынок может быть организован как олигополия Курно, а рынок рекламируемых товаров - как монополистическая конкуренция по ДикситуСтиглицу. Кроме того, как товар, так и контент может быть дифференцирован и вертикально, и горизонтально. Для таких рынков трехсторонняя модель формулируется аналогичным образом с учетом их особенностей. Принцип «трехсторонности», на основе которого строится модель рынка, не зависит от дифференциации товаров и структуры множества фирм, и состоит в том, чтобы учитывать при расчете равновесия взаимовлияние друг на друга всех сторон рынка - и потребителей-аудитории, и медиа, и производителей.

Пусть потребитель-ценополучатель обладает предпочтениями на множестве благ, элементы которого включают в себя как «товарные» элементы (вектор q размерности $n$ ), так и «контентные» (вектор с размерности $m$ ). Потребитель принимает решение в условиях рекламного воздействия, описываемого вектором а. Каждый элемент $a_{i j}$ этого вектора представляет собой долю рекламы «товарного» блага $i=1, \ldots, n$ в «контентном» благе $j=1, \ldots, m\left(\forall j \in\{1, \ldots, m\} a_{1 j}+\cdots+a_{n j} \leq 1\right)$. В общем виде задача, решаемая отдельным потребителем, имеет вид:

$$
u(\mathbf{q}, \mathbf{c}) \rightarrow \max _{(\mathbf{q}, \mathbf{c}) \in X}
$$

где $X=\left\{(\mathbf{q}, \mathbf{c}) \mid \mathbf{p}^{q} \cdot \mathbf{q}+\mathbf{p}^{c} \cdot \mathbf{c} \leq R\right\}-$ бюджетное множество, ограничения которого касаются объемов как товарных, так и на контентных благ, а $\mathbf{p}=\left(\mathbf{p}^{q}, \mathbf{p}^{c}\right)$ - вектор цен на все блага (в том числе $\mathbf{p}^{q}-$ товарные, $\mathbf{p}^{c}-$ контентные), $R$ - общий бюджет потребителя. При построении бюджетного множества для такой задачи важно иметь в виду, что контентные блага имеют ненулевую цену. Цена эта определяется на основе того, во сколько потребителю обходится получение этого блага (например, стоимость номера газеты, где опубликовано контентное благо - статья; стоимость подписки на платный канал, показывающий интересное потребителю кино и т.д.). Другой способ определения цен на контентные блага связан с тем, какую долю времени потребитель тратит на потребление контента. В качестве стоимости контента при таком подходе берется оценка дохода, который мог бы получить потребитель, если бы использовал потраченное на его потребление время для получения дохода.

Полезность потребителя имеет особый вид, связанный с тем, как внедренная в контент реклама влияет на ценность этого контента: 


$$
u(\mathbf{q}, \mathbf{c})=\sum_{i=1}^{n} u_{i}\left(q_{i}\right)\left(1+f_{i}\left(\sum_{j=1}^{m} a_{i j} c_{j}\right)\right)+\sum_{j=1}^{m} v_{j}\left(c_{j}\right)\left(1-g_{j}\left(\sum_{i=1}^{n} a_{i j}\right)\right)
$$

Здесь функции $u_{i}\left(q_{i}\right)$ и $v_{j}\left(c_{j}\right)$ характеризуют полезность потребителя от потребления $q_{i}$ единиц -го товарного и $c_{j}$ единиц $j$-го контентного благ соответственно. Возрастающая функция $g_{j}(\lambda)$ представляет собой меру раздражения потребителя от того, что -е контентное благо на долю $\lambda=\sum_{i=1}^{n} a_{i j}$ состоит из рекламы (например, если на один час вещания канала приходится 50 минут контента и 10 минут рекламы, разделенных между роликами всех сотрудничающих с каналом рекламодателей, то $\left.\lambda=\sum_{i=1}^{n} a_{i j}=1 / 6\right)$. При этом $g(0)=0$, то есть, отсутствие рекламы в данном типе контента позволяет получить от него максимальную полезность. Однако если контент состоит только из рекламы, то его полезность не обязательно равна нулю, так как от просмотра одной лишь рекламы также можно получить определенную пользу или даже удовольствие, поэтому, вообще говоря, $g(1) \leq 1$.

Функция $f_{i}(A)$, также возрастающая, характеризует дополнительную полезность товара $i$, получаемую за счет убеждения рекламой в объеме $A$, который при заданных значениях с и а равен $\sum_{j=1}^{m} a_{i j} c_{j}$ (суммарное количество рекламного контента, связанного с товаром, во всех типах контентных благ). В предлагаемой модели рассматривается преимущественно убеждающая реклама, эффект убеждения которой носит долгосрочный характер и не исчезает после потребления, по крайней мере, в течение какого-то продолжительного периода. Требование убывающей эффективности рекламного убеждения приводит к вогнутости функции $f(\cdot)$, кроме того, $f(0)=0$ : если потребитель не видел рекламы, то его полезность остается «нетронутой». Решение задачи потребителя-ценополучателя при заданном наборе цен р и уровнях рекламы $\mathbf{a}$ порождает спрос двух типов: спрос $\mathbf{Q}(\mathbf{p}, \mathbf{a})$ на товары и $\mathbf{C}(\mathbf{p}, \mathbf{a})$ на контент. Эти функции спроса известны и производителям, и медиафирмам. Так как в описываемой модели фирмы действуют аналогично олигополистам Курно, то для формирования задач производителя и медиафирмы используются обратные функции спроса. Обозначим $\widetilde{\mathbf{p}}^{q}\left(\mathbf{q}, \mathbf{p}_{c}, \mathbf{a}\right)-$ обратную функцию спроса на товары, представляющую собой решение системы уравнений $\mathbf{q}=\mathbf{Q}(\mathbf{p}, \mathbf{a})$. Аналогично определяется обратная функция спроса на контент $\widetilde{\mathbf{p}}^{c}\left(\mathbf{c}, \mathbf{p}_{q}, \mathbf{a}\right)$, решение системы $\mathbf{c}=\mathbf{C}(\mathbf{p}, \mathbf{a})$.

Производители максимизируют прибыль при известном им спросе потребителей, выбирая объемы производства. Обозначим $\mathbf{q}_{i}$ вектор выпусков фирмы $i$, а $\mathbf{a}_{i}-$ вектор, составленный из связанных с ними компонентов вектора рекламных воздействий а. Кроме того, обозначим $c_{k}\left(\mathbf{q}, \mathbf{p}_{c}, \mathbf{a}\right)$ спрос на контент типа $k$, если объем потребления товаров фиксирован на уровнях $\mathbf{q}$, цены на контент равны $\mathbf{p}_{c}$, а доли рекламы в нем определяются вектором $\mathbf{a}$. Задача максимизации прибыли фирмы $i$ имеет вид: 


$$
\max _{\mathbf{q}_{i}, \mathbf{a}_{i}} \pi_{i}^{p}\left(\mathbf{q}_{i}, \mathbf{a}_{i}\right)=\max _{\mathbf{q}_{i}, \mathbf{a}_{i}}\left[\sum_{j \in J_{i}}\left(\widetilde{\mathbf{p}}_{j}^{q}\left(\mathbf{q}, \mathbf{p}_{c}, \mathbf{a}\right) \cdot q_{j}-\sum_{k \in K_{j}} \varphi_{j k}\left(a_{j k} \cdot c_{k}\left(\mathbf{q}, \mathbf{p}_{c}, \mathbf{a}\right), p_{k}^{a}\right)\right)-C_{i}\left(\mathbf{q}_{i}\right)\right]
$$

где $\widetilde{\mathbf{p}}_{i}^{q}$ - составленная из элементов вектор-функции $\widetilde{\mathbf{p}}^{q}(\mathbf{q}, \mathbf{a})$ обратная функция спроса на товары только фирмы $i, J_{i}-$ множество номеров этих товаров, $\mathbf{q}=\left(\mathbf{q}_{i}, i=1,2, \ldots\right)-$ вектор выпусков товаров всех фирм, $K_{j}-$ множество медиа, в которых фирмы размещает рекламу товара $j$, а функция $\varphi_{j k}\left(a_{j k}\right.$. $\left.c_{k}, p_{k}^{a}\right)$ определяется схемой оплаты за размещение рекламы в контенте типа $k$.

Стоимость размещения рекламы зависит от выбранной медиафирмой базовой цены $p_{k}^{a}$ за единицу «рекламной части контента», а также от общего объема рекламы товара в контенте, равной $a_{j k} \cdot c_{k}$. Например, если каждый рекламный контакт обходится фирме в одну и ту же стоимость, то схема оплаты линейная и $\varphi_{j k}\left(a_{j k} \cdot c_{k}, p_{k}^{a}\right)=p_{k}^{a} \cdot a_{j k} \cdot c_{k}$, если каждый дополнительный контакт должен обходиться дороже предыдущего, то $\varphi_{j k}\left(a_{j k} \cdot c_{k}, p_{k}^{a}\right)=p_{k}^{a} \cdot \psi\left(a_{j k} \cdot c_{k}\right)$, где $\psi(\cdot)$ - выпуклая функция, и т.д. При фиксированных ценах на рекламу и контент равновесные объемы производства товара $\mathbf{q}_{i}^{*}\left(\mathbf{p}_{a}, \mathbf{p}_{c}\right)$ и спрос на рекламу $\mathbf{a}_{i}^{*}\left(\mathbf{p}_{a}, \mathbf{p}_{c}\right)$ определяются из равновесия Нэша в возникающей между производителями игре, где выигрышем каждой фирмы служит ее прибыль. Из условия баланса $\mathbf{a}=\mathbf{a}^{*}\left(\mathbf{p}_{a}, \mathbf{p}_{c}\right)$ как решение относительно $\mathbf{p}_{a}$ определяется обратная функция спроса на рекламу $\widehat{\mathbf{p}}^{a}\left(\mathbf{a}, \mathbf{p}_{c}\right)$, подставляя в которую на место цен контента $\mathbf{p}_{c}$ обратную функцию спроса $\widetilde{\mathbf{p}}^{c}(\mathbf{c}, \mathbf{a})$ окончательно получим обратную функцию спроса $\widetilde{\mathbf{p}}^{a}(\mathbf{c}, \mathbf{a})=\widehat{\mathbf{p}}^{a}\left(\mathbf{a}, \widetilde{\mathbf{p}}^{c}(\mathbf{c}, \mathbf{a})\right)-$ уровень цен, по которым в равновесии закупают рекламу производители, если им доступны доли а в контенте объемов с.

Медиафирмы максимизируют прибыль при известном им спросе потребителей на контент и производителей на рекламу. Стратегией медиафирмы является объем производства контента каждого типа и выделяемая в нем общая доля под рекламу. Прибыль $\pi_{j}^{m}$ медиафирмы определяется размером выплат за размещение рекламы, полученных от производителей и совокупной аудиторией произведенного ими медиаконтента. Расходы медиафирмы, в свою очередь, определяются затратами на производство контента. Медиафирма выбирает объем контента, который предлагается аудитории, и выделяет долю своего контента для размещения в ней рекламы производителей. Задача медиафирмы имеет вид:

$$
\max _{\mathbf{a}_{j}, \mathbf{c}_{j}} \pi_{j}^{m}=\max _{\mathbf{a}_{j}, \mathbf{c}_{j}} \sum_{l \in L_{j}}\left[\widetilde{\mathbf{p}}_{l}^{c}(\mathbf{c}, \mathbf{a}) c_{l}+\sum_{i=1}^{n} \varphi_{i l}\left(a_{i l} \cdot c_{l}, \widetilde{\mathbf{p}}_{l}^{a}(\mathbf{c}, \mathbf{a})\right)-C_{l}\left(c_{l}\right)\right],
$$

где $L_{j}-$ множество типов контента, производимого фирмой $j, \widetilde{\mathbf{p}}_{l}^{c}(\mathbf{c}, \mathbf{a})-$ обратная функция спроса на контентное благо $l$ со стороны аудитории, $\widetilde{\mathbf{p}}_{l}^{a}(\mathbf{c}, \mathbf{a})$ - обратная функция спроса на рекламу в этом типе контента, $a_{i l}$ - доля рекламы $i$-го товара в нем, $c_{l}$ - объем производимого контента типа $l$, а $C_{l}\left(c_{l}\right)-$ 
полные затраты на его производство. В качестве равновесных значений объема контента и долей рекламы в нем рассматриваются равновесные по Нэшу стратегии медиафирм в соответствующей игре.

В равновесии объемы контента каждого типа и доли рекламы являются решением задачи медиафирмы, объем товаров и доли рекламы каждого из них в разных типах контента - решением задачи производителя, а спрос на контент и товары - решением задачи потребителя. Следовательно, с точки зрения теоретико-игровой постановки сформулированная модель имеет иерархический характер: потребитель подстраивается под предлагаемые ему медиафирмой и производителем цены на контент и товары. Производители, в свою очередь, выбирают стратегии, зная предлагаемые медиафирмами расценки на рекламу. Медиафирмы находится на самом верху иерархии, первыми выбирая объемы рекламы и контента, реагируя на которые, выбирают свои стратегии производители и потребители-аудитория. С точки зрения такой постановки равновесию на рынке соответствует совершенное по подыграм равновесие в возникающей многошаговой игре.

\section{Трехсторонний рынок: анализ простейших моделей}

Приведем несколько примеров того, как формулируются и исследуются модели трехстороннего рынка. Рассмотрим два типа рынка: двойная монополия и двойная дуополия с горизонтальной дифференциацией товара и контента.

Двойная монополия. В модели рынка с двойной монополией рынок состоит из трех участников - одной фирмы-производителя, одной медиафирмы и репрезентативного потребителя с квазилинейной трансферабельной полезностью. Пусть на рынке существует numeraire-благо, объем его потребления обозначим $y$, и предположим, что общая полезность, получаемая потребителем от потребления набора $(y, c, q)$ имеет вид:

$$
u(y, c, q)=y+2 \sqrt{1+a c} \sqrt{q}+2(1-\lambda a) \sqrt{c}
$$

где $q$ - объем потребления экономического блага, производимого фирмойпроизводителем, $c$ - общий объем потребления медиаконтента, $a$ - доля рекламы экономического блага в медиаконтенте (таким образом, общий объем рекламы, которую видит потребитель, равен $a c)$, а $\lambda \in[0,1]$ - показатель раздражения от рекламы (чем он ближе к 1, тем сильнее реклама снижает полезность медиаконтента). Внутреннее решение задачи потребителя дает следующие значения для функций спроса на контент и товар соответственно:

$$
\begin{gathered}
c\left(a, p_{c}, p_{q}\right)=\frac{(1-\lambda a)^{2}}{\left(p_{c} p_{q}-a\right)^{2}} p_{q}^{2} \\
q\left(a, p_{c}, p_{q}\right)=\frac{1}{p_{q}^{2}}+\frac{a(1-\lambda a)^{2}}{\left(p_{c} p_{q}-a\right)^{2}}
\end{gathered}
$$

Пусть функция оплаты рекламы линейна: $\varphi(A)=A$. Пусть технологии производства и товара, и контента таковы, что медиафирма и фирмапроизводитель обладают постоянной отдачей от масштаба, а предельные из- 
держки их равны $e_{m}$ и $e_{p}$ соответственно. Тогда задача фирмы-производителя примет вид:

$$
\max _{q \geq 0, a \in[0,1]} \pi_{p}=\max _{q \geq 0, a \in[0,1]}\left\{\left(\tilde{p}_{q}\left(q, a, p_{c}\right)-e\right) \cdot q-p^{a} \cdot a \cdot c\left(a, p_{c}, \tilde{p}_{q}\left(q, a, p_{c}\right)\right)\right\} .
$$

Здесь $\tilde{p}_{q}\left(q, a, p_{c}\right)$ - обратная функция спроса, определяемая из решения (5) относительно $p_{q}$. Так как на рынке действует всего один производитель, можно далее в качестве его стратегии рассматривать цену на товар вместо его объема. Оптимальные значения цены $p_{q}^{*}\left(p^{a}, p_{c}\right)$ на товар и спрос $a^{*}\left(p^{a}, p_{c}\right)$ на рекламу в контенте определяются как координаты точки максимума функции:

$$
\pi_{p}\left(p_{q}, a\right)=\left(p_{q}-e_{p}\right) \cdot\left(\frac{1}{p_{q}^{2}}+\frac{a(1-\lambda a)^{2}}{\left(p_{c} p_{q}-a\right)^{2}}\right)-p^{a} \cdot \frac{a(1-\lambda a)^{2}}{\left(p_{c} p_{q}-a\right)^{2}} p_{q}^{2}
$$

При малых значениях цены на рекламу производителю товара выгодно рекламировать, при больших, чем пороговое значение - выгодно не рекламировать и разрушить рекламную цепочку. Функция $\pi_{p}\left(p_{q}, a\right)$ унимодальна по каждому из своих аргументов при фиксированном значении другого. Тем не менее, система уравнений, соответствующая условиям первого порядка для максимума этой функции, нелинейна, а ее решение, хотя и может быть выражено через радикалы, имеет весьма громоздкий вид.

Задача медиафирмы согласно (3) имеет вид:

$$
\max _{a, c} \pi^{m}=\max _{a, c}\left[\tilde{p}_{q}(a, c) c+\tilde{p}^{a}(a, c) \cdot a \cdot c-c\right] \text {. }
$$

Так как она является монополистом, мы можем записать и ее задачу максимизации прибыли относительно цен на рекламу и контент, имея в виду, что выполняются равенства (4) и (5), а $a=a^{*}\left(p^{a}, p_{c}\right)$ представляет собой оптимальный ответ фирмы-производителя на них. Тогда исходную задачу можно переписать в эквивалентном виде относительно цен на контент и рекламу, решение которой в аналитическом виде также крайне громоздко и затруднительно:

$$
\begin{gathered}
\max _{p_{c} \geq 0, p^{a} \geq 0}\left\{\left(p_{c}-e_{m}\right) \cdot c\left(a^{*}\left(p^{a}, p_{c}\right), p_{c}, p_{q}^{*}\left(p^{a}, p_{c}\right)\right)+p^{a} \cdot a^{*}\left(p^{a}, p_{c}\right)\right. \\
\left.\cdot c\left(a, p_{c}, p_{q}^{*}\left(p^{a}, p_{c}\right)\right)\right\}
\end{gathered}
$$

Для поиска решения в численном виде можно использовать любой подходящий пакет прикладных программ². Согласно проведенным численно расчетам, равновесной по Штакельбергу является для медиафирмы стратегия $p^{a} \approx 0.0005, p_{c}=e_{m}$. Наилучшим ответом производителя на нее является пара $p_{q} \approx e_{p}+0.001, a=1$.

Приведенный пример соответствует аудитории, достаточно терпимой к рекламе: даже когда каждая единица контента носит рекламный характер, аудитория получает от него $(1-\lambda) \cdot 100 \%$ исходной, «нерекламной» полезности. Такое отношение позволяет медиафирме «встроить» рекламу в каждую единицу контента ( $a=1)$, за счет чего снизить цену на контент до себестоимости. Почти по себестоимости продавать товар сможет и производитель

\footnotetext{
${ }^{2}$ Автор использовал Waterloo Maple 2019.0
} 
товара $\left(p_{q}\right.$ очень близко к $\left.e_{p}\right)$, при этом реклама обходится ему очень недорого - также за счет высокой толерантности аудитории к ней. При этом за счет низких цен на контент и товары объемы их потребления достаточно высоки, чтобы обеспечить обеим фирмам положительную прибыль.

Двойная дуополия с горизонтальной дифференциаџией товара иконтента. Другим примером может трехсторонней модели может служить модель рынка горизонтально дифференцированного товара и контента. Рассмотрим простую модель: есть ровно два производителя медиа и ровно два производителя товаров. Потребители характеризуются парой $(x, y) \in[0,1] \times[0,1]$, описывающей их предпочтения: $x$ - среди товаров, $y$ - среди контента. Рассматриваемая модель представляет собой двумерный аналог «линейного города» Хотеллинга. Первая медиафирма производит контент типа $x_{0}=0$, вторая - типа $x_{1}=1$; то же справедливо и для производителей товаров. Во избежание путаницы будем обозначать типы контента не цифрами, а буквами: первая медиафирма производит контент типа А $\left(y_{A}=0\right)$, вторая - типа В $\left(y_{B}=1\right)$.

Медиафирма выбирает, по какой цене продавать долю своей аудитории рекламодателям, и по какой цене - контент потребителям. Прибыль ее имеет вид:

$$
\pi_{j}=\left(p_{j}^{c}-c_{m}\right) \cdot\left(D_{0 j}+D_{1 j}\right)+p_{j}^{a}\left(\lambda_{j}^{0} D_{0 j}+\lambda_{j}^{1} D_{1 j}\right)
$$

Здесь: $D_{i j}$ доля потребителей, купивших набор $x_{i}, y_{j}, p_{j}^{c}$ - цена за единицу контента, $p_{j}^{a}$ - цена за один рекламный контакт, $\lambda_{j}^{0}$ - доля рекламы первого производителя в контенте медиафирмы, $\lambda_{j}^{1}$ - доля рекламы второго производителя, $c_{m}^{j}$ - удельные издержки производства контента медиафирмой $j$. Производственная фирма выбирает, по какой цене продавать товар потребителям, и какую долю рекламы в контенте каждого типа приобрести. Прибыль ее имеет вид:

$$
P r_{i}=\left(p_{i}-c_{p}\right) \cdot\left(D_{i A}+D_{i B}\right)-\lambda_{A}^{i} p_{A}^{a}\left(D_{0 A}+D_{1 A}\right)-\lambda_{B}^{i} p_{B}^{a}\left(D_{0 B}+D_{1 B}\right)
$$

Здесь: $p_{i}$ - цена за единицу товара, $\lambda_{j}^{1}$ - для первого производителя, $\lambda_{j}^{2}$ для второго. Каждый потребитель контента типа $j$ видит рекламу обоих товаров, размещенную в нем. Потребитель, приобретающий товар типа $x_{i} \in\{0,1\}$ и контент типа $y_{j} \in\{A, B\}$, получает полезность вида:

$$
u_{x_{i} y_{j}}(x, y)=u_{i j}^{p}-p_{i}-t_{p}\left|x-x_{i}\right|+u_{j}^{m}-p_{j}^{c}-t_{m}\left|y-y_{j}\right|
$$

Здесь: $p_{i}$ цена на товар $x_{i}$ фирмы $i, p_{j}^{c}$ цена на контент $x_{i}$ медиафирмы $j$, $u_{i j}^{p}$ и $u_{j}^{m}$ есть резервные цены потребителей на товары и медиаконтент соответственно, определяемые рекламным воздействием фирм. Параметры $t_{p}$ и $t_{m}$ характеризуют уровень дифференциации товара и контента соответственно, они также зависят от уровней $\lambda_{j}^{i}$ рекламы фирм в различных типах контента. Учесть убеждающее влияние рекламы возможно с помощью двух способов, 
родственных тем, что были использованы в [20, 21]. В первом случае реклама увеличивает готовность потребителя платить за товар, и в модели меняется резервная цена $u_{i j}^{p}=u^{p}\left(1+\lambda_{j}^{i}\right)^{\gamma}, \gamma \in(0,1]$. Во втором случае реклама может усиливать дифференциацию товара, и $t_{p}=t_{p}\left(1+\alpha_{0}+\alpha_{1}+\beta_{0}+\beta_{1}\right)$. Раздражение от рекламы влияет на резервную цену рекламы, как и в других трехсторонних моделях: $u_{j}^{m}=u^{m}\left(1-\left(\lambda_{j}^{0}+\lambda_{j}^{1}\right)\right)^{\delta}, \delta \in(0,1]$.

Взаимодействие участников рынка происходит в несколько этапов. Сначала медиафирмы определяют цены на рекламу, затем на контент. Далее вступают производители: они выбирают рекламные стратегии, после чего цену на свой товар. Далее потребители выбирают свой набор из товара и контентного блага. Как и в случае с Курно-подобной моделью, равновесию на описываемом рынке соответствует совершенное по подыграм равновесие в описанной игре.

Для простейшего случая $(\gamma=\delta=1)$ с повышающей готовность платить рекламой было проведено численное исследование множества совершенных подыгровых равновесий. Равновесие в этом случае имеет «разделяющий» и симметричный вид, при этом убеждающие воздействия фирм «гасят» друг друга, что соответствует результату [20]. Более точно, каждая из фирм в равновесии закупает рекламу только у одной медиафирмы, таким образом осуществляя рекламное воздействие только на ее аудиторию. При этом доли рекламы, закупаемые у каждой из медиафирм оказываются равными. Медиафирмы, в свою очередь, выбирают одинаковые цены на рекламу. Такой исход на рынке соответствует достаточно часто встречающейся на практике ситуации, при которой медиа стараются максимально сделать свой контент как можно более уникальным и непохожим на контент конкурентов, становясь в наибольшей степени нишевыми. Рекламодатели же, в свою очередь, предпочитают закупать рекламу у тех медиа, которые обладают наибольшим показателем аффинитивности у своей целевой аудитории. При этом для конечных потребителей ситуация цена на товары оказывается такой же, как и на обычном рынке с горизонтальной дифференциацией а la Хотеллинг.

\section{Выводы}

Проведенный анализ современных взглядов на экономические эффекты интеграции рынков медиа и промышленности позволил сделать следующие выводы. С одной стороны, имеет место значительное и многоплановое воздействие медиа на структуру рынков промышленных товаров и исход взаимодействия их участников через рекламу как основной инструмент управления спросом на них. Помимо экономической теории рекламы, предполагающей ее одним из инструментов фирм-производителей, в последние годы значительное развитие получила и экономическая теория медиа. Для медиа реклама является одним из существенных источников дохода, поэтому рыночная стратегия медиафирмы, как правило, включает в себя и «контентную», и «рекламную» часть. Контент представляет собой товар, удовлетворяющий потребности аудитории, а реклама - рекламодателей, что естественным образом приводит к модели медиарынка как двустороннего рынка с медиафирма- 
ми в роли платформ. Подобные модели позволяют изучить многие эффекты, в том числе сетевые, связанные с формированием цен на рекламу и контент, однако не дают понимания, как ситуация на медиарынке влияет на ценообразование в «товарном» секторе экономики. Данная задача усложняется еще сильнее, если вспомнить, что потребителями рекламируемых товаров и аудиторией контента медиа являются одни и те же индивиды.

Для того, чтобы учесть наличие трех сторон рынка - производителейрекламодателей, медиафирмы, производящие контент и доводящие рекламу до аудитории, и потребителей, в настоящей работе была предложена методология построения моделей нового типа - моделей трехстороннего рынка. Подобные модели могут описывать рынок с любой структурой множества фирм и любым типом дифференциации товара. Принцип «трехсторонности», лежащий в основе предложенной методологии, состоит в том, чтобы учитывать при расчете равновесия взаимовлияние друг на друга всех сторон рынка - и потребителей-аудитории, и медиа, и производителей.

Как показывают исследованные в работе примеры, трехсторонние модели даже в наиболее простых предположениях позволяют смоделировать достаточно сложные эффекты межотраслевого влияния медиаиндустрии и производственных фирм. В частности, на рынках с двойной монополией (единственная медиафирма, единственный производитель) обнаруживает «засорение» рекламой каждой единицы контента, за счет чего цена на него снижается до себестоимости. При этом и монополист-производитель сможет опустить цену на свой товар значительно ниже «обычного» монопольного уровня почти до себестоимости - за счет возрастания спроса на него из-за рекламы. При этом сама реклама обходится ему очень недорого - также за счет определенной терпимости к ней аудитории. При этом за счет низких цен на контент и товары объемы их потребления достаточно высоки, чтобы обеспечить обеим фирмам положительную прибыль. Если же на рынке действуют хотя бы по две компании - как медийных, так и производственных - и возникает дифференциация и товара, и контента, то в соответствующей модели существует равновесие с «кооперацией» медиафирм и производителей. Медиафирмы назначают одинаковые цены на рекламу, а каждый производитель покупает рекламу только у одной из них. Таким образом, будучи независимыми компаниями, производители и медиафирмы разбиваются на «пары», фактически формирующие маркетинговые каналы.

\section{Заключение}

В настоящей работе кратко перечислены предпосылки к построению и приведены примеры трехсторонних моделей рынков с участием производителей, потребителей и медиафирм, выступающих не только как посредникиплатформы, но и как производители товара особого типа (контента, коммуникационных услуг). Анализ общих условий существования и свойств равновесий, возникающих в моделях подобного типа, представляют собой актуальные и пока нерешенные задачи, решение которых позволит дать ответ на ранее не исследованные новые вопросы экономической теории медиа и рекламы, а также понять теоретико-стратегические аспекты межотраслевого взаимодействия, связывающего медиаиндустрию с другими отраслями эко- 
номики. Дальнейшее теоретическое развитие моделей трехсторонних рынков может проходить по множеству направлений. Во-первых, перед исследователем стоит вопрос об условиях существования в ней равновесий и их количества (ситуация отсутствия равновесия для некоторых функций полезности и стоимости рекламы более чем вероятна). Во-вторых, требуют полноценного анализа модификации трехсторонней модели для рынков несовершенной конкуренции (монополистическая конкуренция, олигополия на медийной и производственной сторонах рынка, модели рынков с фирмами-лидерами) с различными типами дифференциации товара (вертикальной, горизонтальной). Третье направление исследования представляют собой задачи сравнительной статики для описания реакции равновесных цен на шоки параметров модели (например, на изменение характера реакции потребителя на рекламу). Кроме того, встает отдельная группа вопросов, связанная с анализом трехсторонних рынков в долгосрочном периоде, включающая в себя как определение структуры рынка при условии свободного входа на него фирм каждого типа (медиа и производственных), так и анализ динамики рынка с фиксированной структурой (устойчивость равновесий, (не)сходимость при времени, стремящемся к бесконечности и т.д.).

\section{Список источников / References}

1. Dunas D.V., Vartanov S.A. (2020). Emerging digital media culture in Russia: modeling the media consumption of generation Z. Journal of Multicultural Discourses, 2020, DOI: 10.1080/17447143.2020.1751648

2. Дунас Д.В., Вартанов С.А., Кульчицкая Д.Ю., Салихова Е.А., Толоконникова А.В. Теоретические аспекты изучения медиапотребления российской молодежи: к пересмотру теории использования и удовлетворения. Вестник Московского университета, 2019, серия 10: Журналистика, № (2), сс. 3-28.

3. Bagwell K. The economic analysis of advertising. Handbook of industrial organization, 2007, vol. 3, pp. 1701-1844.

4. Motta M. Advertising bans. SERIEs - Journal of the Spanish Economic Association, 2013, vol. 4 (1), pp. 61-81.

5. Sethi S.P. Deterministic and stochastic optimization of a dynamic advertising model. Optimal Control Applications and Methods, 1983, vol. 4 (2), pp. 179-184.

6. Weber T.A. Infinite-horizon optimal advertising in a market for durable goods. Optimal Control Applications and Methods, 2005, vol. 26 (6), pp. 307-336.

7. Ishigaki H. Informative advertising and entry deterrence: a Bertrand model. Economics Letters, 2000, vol. 67 (3). pp. 337-343.

8. Kuzmanović M., Kovačević-Vujčić V., Martić M. Three-stage entry game: the strategic effects of advertising. Yugoslav Journal of Operations Research, 2011, vol. 21 (2), pp. 163-185.

9. Tirole J. The theory of industrial organization. MIT press, 1988.

10. Jørgensen S., Zaccour G. A survey of game-theoretic models of cooperative advertising. European Journal of Operational Research, 2014, vol. 237, no. 1, pp. 1-14.

11. Rochet J.C., Tirole J. Platform competition in two-sided markets. Journal of the European economic association, 2003, vol. 1 (4), pp. 990-1029.

12. Anderson S.P., Gabszewicz J.J. The media and advertising: a tale of two-sided markets. Handbook of the Economics of Art and Culture, 2006, vol. (1), pp. 567-614. 
13. Баландина М.С., Баскакова И.В. Двусторонние рынки: определение понятия, ключевые характеристики и инструменты оценки. Известия Уральского государственного экономического университета, 2016, №2 (64), сс. 12-20.

14. Шаститко А.Е., Паршина Е.Н. Рынки с двусторонними сетевыми эффектами: спецификация предметной области. Современная конкуренщия, 2016, т. 10 (1), cc. 5-18.

15. Godes D., Ofek E., Sarvary M. Content vs. advertising: The impact of competition on media firm strategy. Marketing Science, 2009, vol. 28 (1), pp. 20-35.

16. Prat, A. and Valletti, T. M. Attention oligopoly. Mimeo, 2018.

17. Reisinger M. Platform competition for advertisers and users in media markets. International Journal of Industrial Organization, 2012, vol. 30 (2), pp. 243-252.

18. Chatterjee P., Zhou B. Sponsored Content Advertising in a Two-sided Market. Mimeo, 2015.

19. Вартанов С.А. Динамика развития медиаиндустрии России в 2000-2014 гг.: общие тренды и взаимосвязь с макроэкономическими показателями. Медиаскоп (электронный журнал), 2015, т. 3. Available at: http://www.mediascope.ru/1831 (accessed 11.04.2020).

20. Bloch F., Manceau D. Persuasive advertising in Hotelling's model of product differentiation. International Journal of Industrial Organization, 1999, vol. 17 (4), pp. 557-574.

21. Von der Fehr N. H. M., Stevik K. Persuasive advertising and product differentiation. Southern Economic Journal, 1998, vol. 65 (1), pp. 113-126.

\section{Сведения об авторе / About author}

Вартанов Сергей Александрович, кандидат физико-математических наук, доцент кафедры эконометрики и математических методов экономики Московской школы экономики, Московский государственный университет имени М.В. Ломоносова. 119234 Россия, Москва, Ленинские Горы, д.1, стр. 61

E-mail:sergvart@gmail.com

Sergey A. Vartanov, Candidate of Physical and Mathematical Sciences, Associate Professor at the Chair of Econometrics and Mathematical Methods in Economics, Moscow School of Economics, Lomonosov Moscow State University. Bldg. 61, 1 Lenin Hills, Moscow, Russia 119234.

E-mail: sergvart@gmail.com 